\title{
ANALYSIS AND DESIGN OF DRAINAGE SYSTEM PLANNING STUDY (CASE STUDY GRAHA WISATA SIDOARJO RESIDENTIAL)
}

\author{
M. Fachrul Reza, \\ Faculty of Engineering Narotama University Surabaya, \\ fachrulreza1997@gmail.com \\ Diah Ayu Restuti Wulandari \\ Faculty of Engineering, Narotama University Surabaya,
}

\begin{abstract}
Graha Wisata Sidoarjo Housing has a land area of $470,000 \mathrm{~m} 2$ located in Lebo Village, Sidoarjo District, Sidoarjo Regency. The housing is passed by the Anak Afvoer Sidokare channel. With the change in land function, it is necessary to analyze the capacity of the Anak Afvoer Sidokare watershed. The rainfall data used is the annual maximum rainfall from the rain station Durungbedug, Ketintang, Sidoarjo, Sumput .. The method of rainfall analysis uses the Log Pearson type III method. Planned flood discharge in the Anak Afvoer Sidokare watershed at 25 years using the Nakayasu method is $450.55 \mathrm{~m} 3 / \mathrm{sec}$ while the existing Afvoer capacity with dimensions of $10 \mathrm{~m} \times 4.172 \mathrm{~m}$ is $537.39 \mathrm{~m} 3 / \mathrm{sec}$, the cross section of the Son Afvoer Sidokare channel is not overflow.
\end{abstract}

Keywords: flood, drainage system, capacity.

\section{INTRODUCTION}

Graha Wisata Sidoarjo Housing is a housing that is above the rice fields and passed by the Anak Afvoer Sidokare channel. With the transition of land functions into settlements. or changes in land use, it is necessary to analyze the Anak Afvoer Sidokare watershed. In determining the planned flood discharge there are two things that need to be considered, namely overflow or no overflow.

\section{Floods}

Flooding is an indication of an imbalance in the environmental system in the process of flowing water and is influenced by the amount of water flowing that exceeds the drainage capacity (Suripin, 2004).

To get the flood design there are two approaches, namely the hydrograph method and the non-hydrograph hifrograph method producing the magnitude of the flood each peak hour. An example of the method used is Nakayasu synthetic unit hydrograph. 


\section{Location of the study}

The research location used for the study was the Anak Afvoer Sidokare river, Sidoarjo district, Sidoarjo regency

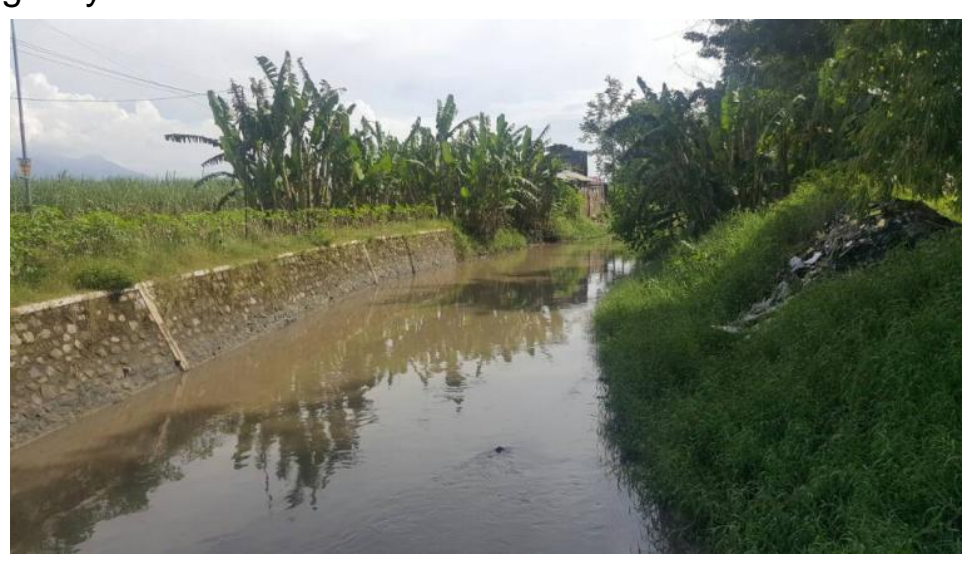

Figure 1. Condition of the Afvoer Sidokare River

Data requirements include rainfall data for 10 years from 2006 - 2016 at the rain station Durungbedug, Ketintang, Sidoarjo, Sumput.

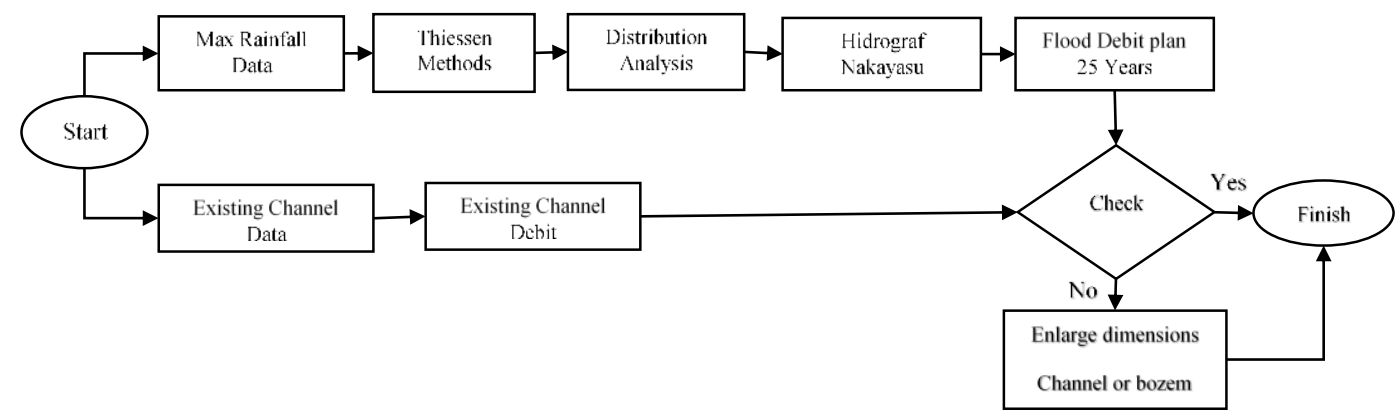

Figure 2. Data requirements include rainfall data for 10 years from 2006- 2016

\section{METHODOLOGY}

\section{Rain Intensity}

Rain that occurs in each area must be different from the period of time, namely annual rainfall, monthly rainfall and daily rainfall. The results obtained are used to determine future prospects.

To calculate the intensity of the rain, the mononobe formula is used:

$I=\frac{R 24}{24}\left(\frac{24}{t}\right)^{\frac{2}{3}}$

With

I = Rain Intensity ( $\mathrm{mm} /$ hour)

$\mathrm{t}=$ The duration of rain (hours)

R24 = Daily maximum rainfall in 24 hours $(\mathrm{mm})$ 
Table 1. Distribution of rain every hour

\begin{tabular}{|r|r|r|r|r|r|r|}
\hline \multirow{3}{*}{ Waktu } & \multicolumn{7}{|c|}{ Periode Ulang } \\
\cline { 2 - 7 } & 2 & 5 & 10 & 25 & 50 & 100 \\
\cline { 2 - 7 } & 94.6452 & 121.228 & 142.038 & 172.223 & 197.623 & 225.684 \\
\hline 1 & 32.8117 & 42.0273 & 49.2418 & 59.7063 & 68.5122 & 78.2402 \\
\hline 2 & 20.67 & 26.4755 & 31.0204 & 37.6126 & 43.16 & 49.2882 \\
\hline 3 & 15.7742 & 20.2046 & 23.673 & 28.7038 & 32.9372 & 37.614 \\
\hline 4 & 13.0213 & 16.6785 & 19.5416 & 23.6944 & 27.1891 & 31.0496 \\
\hline 5 & 11.2214 & 14.3731 & 16.8405 & 20.4193 & 23.4308 & 26.7578 \\
\hline 6 & 9.93712 & 12.7281 & 14.9131 & 18.0822 & 20.7492 & 23.6953 \\
\hline 7 & 8.96663 & 11.485 & 13.4566 & 16.3163 & 18.7227 & 21.3812 \\
\hline 8 & 8.20291 & 10.5068 & 12.3105 & 14.9266 & 17.1281 & 19.56 \\
\hline 9 & 7.58344 & 9.71337 & 11.3808 & 13.7993 & 15.8346 & 18.0829 \\
\hline 10 & 7.06906 & 9.05451 & 10.6088 & 12.8633 & 14.7605 & 16.8563 \\
\hline 11 & 6.63386 & 8.49708 & 9.95571 & 12.0714 & 13.8518 & 15.8186 \\
\hline 12 & 6.25999 & 8.01821 & 9.39464 & 11.3911 & 13.0711 & 14.9271 \\
\hline 13 & 5.93471 & 7.60156 & 8.90646 & 10.7992 & 12.3919 & 14.1515 \\
\hline 14 & 5.64862 & 7.23513 & 8.47713 & 10.2786 & 11.7946 & 13.4693 \\
\hline 15 & 5.3947 & 6.90988 & 8.09605 & 9.81655 & 11.2644 & 12.8638 \\
\hline 16 & 5.16751 & 6.61889 & 7.7551 & 9.40315 & 10.79 & 12.3221 \\
\hline 17 & 4.96282 & 6.35671 & 7.44792 & 9.03068 & 10.3626 & 11.834 \\
\hline 18 & 4.77727 & 6.11904 & 7.16945 & 8.69304 & 9.97515 & 11.3915 \\
\hline 19 & 4.60814 & 5.90241 & 6.91563 & 8.38528 & 9.622 & 10.9882 \\
\hline 20 & 4.45323 & 5.70398 & 6.68314 & 8.10339 & 9.29854 & 10.6188 \\
\hline 21 & 4.31071 & 5.52144 & 6.46926 & 7.84405 & 9.00095 & 10.279 \\
\hline 22 & 4.17907 & 5.35283 & 6.27171 & 7.60451 & 8.72609 & 9.9651 \\
\hline 23 & 4.05704 & 5.19652 & 6.08857 & 7.38246 & 8.47129 & 9.67412 \\
\hline 24 & 3.94355 & 5.05116 & 5.91825 & 7.17594 & 8.23431 & 9.40349 \\
\hline & & & & & & \\
\hline
\end{tabular}

\section{Nakayasu Method}

Watershed data parameters

$$
\begin{array}{ll}
\text { Large } & =16,8 \mathrm{~km}^{2} \\
\text { River large }=20 \mathrm{~km} & \\
\text { Average Slope } & =0,0002 \\
\text { Coefisien } & =2
\end{array}
$$

\section{Paraneter Tg}

$\mathrm{Tg}=0,4+(0,058 . \mathrm{L})$

$\mathrm{Tg}=1,56$ hours

\section{Parameter $\mathrm{Tr}$}

$\mathrm{Tr}=0,75 \mathrm{Tg}$

$\mathrm{Tr}=1,17$ hours

\section{Parameter Tp}

$\mathrm{Tp}=\mathrm{Tg}+0,8 \mathrm{Tr}$

$\mathrm{Tp}=2,496$ hours

\section{Parameter T0,3}

$$
\begin{array}{ll}
\mathrm{T} 0,3 & =\alpha \cdot \mathrm{Tg} \\
\mathrm{T} 0,3 & =2 \cdot 1,56=3 \cdot 12 \text { hours } \\
\mathrm{Tp}+\mathrm{T} 0,3 & =2,496+3,12=5,616 \text { hours } \\
\mathrm{Tp}+\mathrm{T} 0,3+1,5 . \mathrm{T} 0,3=5,615+1,5 \cdot 3,12=10,296 \text { hours }
\end{array}
$$

$$
\begin{aligned}
& \text { Parameter } \mathbf{Q p} \\
& =\frac{A \cdot R o}{3,6(0,3 \text { tp }+T 0,3)} \\
& =\frac{16,8 \cdot 1}{3,6(0,3 \cdot 2,496+10,296)}=1,206 \mathrm{~m}^{3} / \mathrm{sec}
\end{aligned}
$$

Ordynat hydrograf
1.
$0<\mathrm{t}<\mathrm{Tp}$
$=>0<t<2,496$ 
$\mathrm{Qt}=\mathrm{Q} \max (\mathrm{t} / \mathrm{Tp}) 2,4$

2. $\quad T p<t<(T p+T 0,3)$

$=>2,496<\mathrm{t}<5,616$

Qt $=Q \max (0,3)(\mathrm{t}-\mathrm{Tp} / \mathrm{T} 0,3)$

3. $\quad(\mathrm{Tp}+\mathrm{T} 0,3)<\mathrm{t}<\mathrm{Tp}+\mathrm{T} 0,3+1,5 . \mathrm{T} 0,3$

Qt $=Q \max (0,3)((t-T p)+0.5 T 0,3) / 1,5 T 0,3)$

4. $\quad t>(T p+T 0,3+1,5 . T 0,3)$

$=>5,616<t<10,296$

$\mathrm{Qt}=\mathrm{Q} \max (0,3)((\mathrm{t}-\mathrm{Tp})+1,5 \mathrm{~T} 0,3) / 2 \mathrm{~T} 0,3)$

Table 2. Ordynat Hydrograf

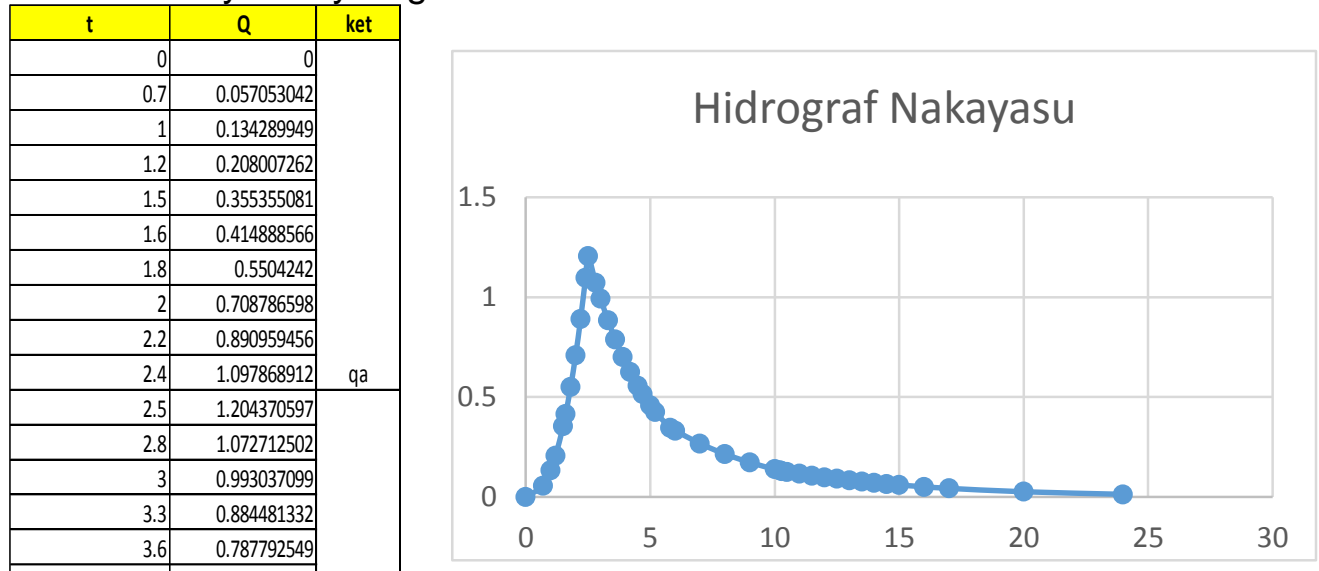

Figure 3. Nakayasu

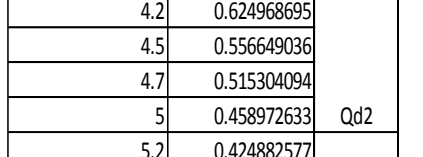$$
=>t>10,296
$$

\begin{tabular}{|c|c|c|}
\hline 5.2 & 0.424882577 & \\
\hline 5.8 & 0.347692779 & \\
\hline 6 & 0.332912699 & \\
\hline 7 & 0.267919186 & \\
\hline 8 & 0.215614154 & \\
\hline 9 & 0.173520471 & \\
\hline 10 & 0.139644608 & \\
\hline 10.2 & 0.133708452 & $q^{3}$ \\
\hline 10.3 & 0.130864103 & \\
\hline 10.5 & 0.126669353 & \\
\hline 11 & 0.116761347 & \\
\hline 11.5 & 0.10762834 & \\
\hline 12 & 0.099209711 & \\
\hline 12.5 & 0.091449583 & \\
\hline 13 & 0.084296448 & \\
\hline 13.5 & 0.077702827 & \\
\hline 14 & 0.071624955 & \\
\hline 14.5 & 0.066022491 & \\
\hline 15 & 0.060858249 & \\
\hline 16 & 0.05171 & \\
\hline 17 & 0.043936922 & \\
\hline 20 & 0.026952242 & \\
\hline 24 & 0.014048024 & q4 \\
\hline
\end{tabular}

Calculation of the 25-year return flood rate with the maximum Nakayasu method occurred at $450.56 \mathrm{~m} 3 / \mathrm{sec}$. For detailed calculations can be seen in table 7 
Table 3. Flood Discharge On 25 Years Return

\begin{tabular}{|c|c|c|c|c|c|c|c|c|c|c|c|c|c|c|c|c|c|c|c|c|c|c|c|c|c|c|}
\hline \multirow[t]{2}{*}{$\mathrm{m}$} & & \multicolumn{24}{|c|}{ Q Akibat Hujan Netto } & \\
\hline & & 9.71 & $37.61 \mid$ & 28.70 & $23.69 \mid$ & 20.42 & \begin{tabular}{|l|l|}
18.08 \\
\end{tabular} & 16.32 & 14.93 & 13.80 & 12.86 & 12.07 & 11.39 & 10.80 & 10.28 & 9.82 & 9.40 & 9.03 & 8.69 & 8.39 & 8.10 & 7.84 & 7.60 & \begin{tabular}{|l|}
7.38 \\
\end{tabular} & 7.18 & \\
\hline 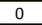 & 0 & 0 & 0 & 0 & 0 & 0 & 0 & & 0 & \begin{tabular}{l|l}
0 & \\
\end{tabular} & 0 & 0 & 0 & 0 & 0 & 0 & 0 & & & & & & & & & \\
\hline .7 & & & & & & & & & & 0.79 & & & & & & 0.56 & & 0.5 & & & & 45 & 0.43 & 0.42 & & \\
\hline \pm & & & & & & & & & & & 73 & 62 & & & & 32 & & & & & & & & & & \\
\hline & & & & & & & & & & 77 & & & & & & & 6 & & & & & & & & & \\
\hline & & & & & & & 6. & & & 4.90 & 57 & & & 84 & & & & & & & & & & & & \\
\hline & & & & & & & 7.50 & & & 5.73 & & & & & & & & & & & & 25 & & & & .2 \\
\hline 1.8 & & & & & & & 9.95 & 98 & & 7.60 & & & & & & 5.40 & & & & & & & & & & \\
\hline & & & & 34 & & & 12.82 & 56 & 58 & 9.78 & 12 & 56 & 07 & 65 & .29 & 96 & 66 & & 6.16 & & & & & & & 5.1 \\
\hline 2.2 & & & & & & & & & & & & & & & 9.1 & 8.75 & & & & & & & & & & \\
\hline & & & & 51 & 01 & 42 & & 91 & 39 & 15.15 & 12 & 13.25 & 12.51 & 11.86 & 11.28 & 10.78 & .32 & & 9.54 & 21 & & & & & & 0.7 \\
\hline & & & & & & & & & & & & 14.54 & 13.72 & & & & & & & & & & & & & \\
\hline & & & & & & & & & 6.01 & & 80 & 95 & 12.22 & 58 & 11.03 & 0.53 & 1.09 & 59 & 9.33 & 8.99 & 69 & 3.41 & 8.16 & .92 & 70 & 1.31 \\
\hline 3 & & & & & & & & & & & & & & & & & & & & & & & & & & \\
\hline 3.3 & & & & 39 & 96 & 06 & 5.99 & 43 & 3.20 & 12.21 & 1.38 & 10.68 & 0.08 & .55 & 1.09 & 8.68 & .32 & 99 & 7.69 & 7.42 & .17 & 5.94 & 6.73 & 6.53 & 35 & 0.8 \\
\hline & & & & & & & & & & & & & & & & & & & & & & & & & & \\
\hline 3.9 & & & & 14 & & & 2.69 & 45 & 0.47 & 9.68 & .03 & 47 & 7.99 & 7.58 & 7.21 & 6.89 & 5.60 & 34 & 6.10 & 5.88 & 69 & 50 & 5.34 & 5.18 & 04 & 2.5 \\
\hline & & & & & & & & & & & & & & & & & & & & & & & & & & \\
\hline 4.5 & & & & 98 & & .37 & .07 & .08 & 3.31 & 68 & .116 & 72 & 34 & 01 & 172 & 5.46 & 23 & & 4.84 & & 51 & .37 & 4.23 & 4.11 & & 8.2 \\
\hline & & & & & & & & & & & & & & & & & & & & & & & & & & \\
\hline 5 & & & & & & & 30 & 49 & 6.85 & 33 & 90 & 54 & 5.23 & 4.96 & 4.72 & 4.51 & 32 & & 3. & & & 0 & 3.4 & 3.36 & & 1.7 \\
\hline & & & & & & & & & & & & & & & & & & & & & & & & & & \\
\hline 5.8 & & & & & & .10 & 6.29 & 67 & 5.19 & 4.80 & 4.47 & 20 & 3.96 & 3.75 & 3.57 & 3.41 & & & & & 82 & & 2.6 & 2.5 & & 0.0 \\
\hline & & & & & & & & & & & & & & & & & & & & & & & & & & \\
\hline 7 & & & & & 35 & & 4.84 & .37 & 4.00 & 3.70 & .45 & 23 & .05 & 2.89 & 2.75 & 2.63 & & & & & 17 & & & & & 0.2 \\
\hline & & & & & & & & & & & & & & & & & & & & & & & & & & \\
\hline 9 & & & & & & & & & & & & & & & & & & & & & & & & & & \\
\hline 16 & & & & & & & & & & & & & & & & & & & & & & & & & & 24 \\
\hline & & & & & & & & & & & & & & & & & & & & & & & & & & \\
\hline & & & & & & & & & & & & & & & & & & & & & & & & & & \\
\hline & & & & & & & & & & & & & & & & & & & & & & & & & & 3 \\
\hline & & & & & & & & & & & & & & & & & & & & & & & & & & \\
\hline & & & & & & & & & & & & & & & & & & & & & & & & & & \\
\hline & & & & & & & & & & & & & & & & & & & & & & & & & & \\
\hline & & & & & & & & & & & & & & & & & & & & & & & & & & \\
\hline & & & & & & & & & & & & & & & & & & & & & & & & & & \\
\hline & & & & & & & & & & & & & & & & & & & & & & & & & & \\
\hline & & & & & & & & & & & & & & & & & & & & & & & & & & \\
\hline 14 & & & & & & & & & & & & & & & & & & & & & & & & & & \\
\hline & & & & & & & & & & & & & & & & & & & & & & & & & & \\
\hline 16 & & & & & & & & & & 0.7 & & & & & & & & & 0.4 & & & & & & & \\
\hline & & & & & & & & & & & & & & & & & & & & & & & & & & \\
\hline 20 & & & & & & & & & & 0.3 & 0.35 & & & 0,29 & 0.2 & & & & 0.23 & 023 & .22 & 0.21 & 0.20 & .20 & 19 & \\
\hline & & & & & & & & & & & & & & & & & & & & & & & & & & \\
\hline
\end{tabular}

Afvoer cross section calculation

$\begin{array}{ll}\text { Channel Name } & : \text { Afvoer Sidokare } \\ \text { Length } & : 20 \mathrm{~km} \\ \text { Wide (b) } & : 10 \mathrm{~m} \\ \text { High (h) } & : 4,172 \mathrm{~m} \\ \text { Slope of the wall }(\mathrm{m}) & : 4,66 \mathrm{~m} \\ \text { Elevated headwaters } & : 12 \mathrm{~m} \\ \text { Elevated downstream } & :-11 \mathrm{~m} \\ \text { Slope Of Channel(s) } & : 0,0011 \\ \mathrm{~K} & : 70 \\ \mathrm{M} & : 5\end{array}$
Sectional of area
$:(\mathrm{B}+\mathrm{mh}) \mathrm{h}=(10+4 \cdot 66 \times 4 \cdot 172) \cdot 4.172=122,90 \mathrm{~m} 2$

Wet circumference $(P): B+2 h\left(m^{2}+1\right) 0.5=10+2.4,172 .((4,662)+1) 0,5=49,80 M$

Hydraulic finger $(R) \quad: A / P=2,50 \mathrm{M}$

Flow Speed $\quad: K \times R^{\wedge}(2 / 3) \times S^{\wedge}(1 / 2)=4,37261 \mathrm{~m} / \mathrm{sec}$

Channel Discharge $\quad$ : V.A $=4,37.122 .9=537,393 \mathrm{~m} 3 / \mathrm{sec}$

\section{RESULTS AND DISCUSSION}

\section{Hydrological Analysis}

Hydrological analysis was carried out to determine the rainfall intensity of the data used in the form of rainfall data for the last 10 years from 2006 to 2016. From the data then determine the analysis of frequency distribution and design flood discharge. 


\section{Maximum rainfall analysis}

Through data from the Sidoarjo Regency Water Service in the Anak Afvoer Sidokare Watershed, there are 4 rain gauge stations, namely Durungbedug Station $(4.20 \mathrm{~km} 2)$, Ketintang Station (4.05 km2) Sidoarjo Station (2.35 km2) and Sumput Station (3.55 km2)

Table 4. Maximum rainfall data

\begin{tabular}{|r|c|r|r|r|r|r|}
\hline \multirow{2}{*}{ TAHUN } & \multirow{2}{*}{ TANGGAL } & \multicolumn{4}{|c|}{ STASIUN PENAKAR HUJA } & \multirow{2}{*}{ TOTAL } \\
\cline { 3 - 6 } & & DURUNGBEDUG & \multicolumn{1}{|c|}{ KETINTANG } & \multicolumn{1}{c|}{ SIDOARJO } & \multicolumn{1}{c|}{ SUMPUT } \\
\cline { 3 - 6 } & & $\mathbf{0 . 2 9 7}$ & $\mathbf{0 . 2 8 6}$ & $\mathbf{0 . 1 6 6}$ & $\mathbf{0 . 2 5 1}$ & \\
\hline 2006 & 26 Peb & 115 & 25 & 80 & 104 & 80.668 \\
\hline 2007 & 8-Mar & 86 & 75 & 78 & 76 & 79.014 \\
\hline 2008 & 31-Jan & 105 & 115 & 110 & 95 & 106.184 \\
\hline 2009 & 6-Mar & 170 & 90 & 71 & 13 & 91.272 \\
\hline 2010 & $15-$-Oct & 143 & 125 & 168 & 160 & 146.265 \\
\hline 2011 & 26-Dec & 141 & 75 & 0 & 141 & 98.693 \\
\hline 2012 & 15-Jan & 68 & 85 & 53 & 115 & 82.166 \\
\hline 2013 & 2-Jan & 122 & 81 & 96 & 135 & 109.208 \\
\hline 2014 & 13-Mar & 113 & 129 & 18 & 74 & 92.018 \\
\hline 2015 & 19-Mar & 100 & 80 & 0 & 77 & 71.898 \\
\hline 2016 & 10-Oct & 176 & 176 & 170 & 167 & 172.746 \\
\hline
\end{tabular}

\section{Frequency Analysis}

The selection of the appropriate type of rainfall distribution is based on the values of asymmetric coefficients (Cs), coefficient of variation (Cv) and coefficient of kurtosis (Ck). The coefficient is obtained by determining the value of statistical parameters from rainfall data

Table 5. Frequency Analysis

\begin{tabular}{|c|c|c|c|c|c|c|c|}
\hline No & Tahun & Tanggal & $\mathrm{Xi}$ & $\mathrm{Xi}-\mathrm{Xr}$ & $\mathrm{Xi}-\mathrm{Xr} 2$ & $\mathrm{Xi}-\mathrm{Xr} 3$ & $\mathrm{Xi}-\mathrm{Xr} 4$ \\
\hline 1 & 2015 & 19-Mar & 71.898 & $\begin{array}{r}-30.842 \\
\end{array}$ & 951.2063 & -29337 & 904793.3418 \\
\hline 2 & 2007 & 8-Mar & 79.014 & -23.725 & 562.8768 & -13354 & 316830.2562 \\
\hline 3 & 2006 & $26 \mathrm{Peb}$ & 80.668 & -22.071 & 487.1429 & -10752 & 237308.2 \\
\hline 4 & 2012 & 15-Jan & 82.166 & -20.573 & 423.2516 & -8707.6 & 179141.9563 \\
\hline 5 & 2009 & 6-Mar & 91.272 & -11.467 & 131.4938 & -1507.8 & 17290.61315 \\
\hline 6 & 2014 & 13-Mar & 92.018 & -10.721 & 114.9504 & -1232.4 & 13213.58505 \\
\hline 7 & 2011 & 26-Dec & 98.693 & -4.047 & 16.3748 & -66.262 & 268.1340912 \\
\hline 8 & 2008 & 31-Jan & 106.184 & 3.445 & 11.8652 & 40.8707 & 140.7825221 \\
\hline 9 & 2013 & 2-Jan & 109.208 & 6.469 & 41.8521 & 270.755 & 1751.60076 \\
\hline 10 & 2010 & 15-Oct & 146.265 & 43.526 & 1894.5004 & 82459.8 & 3589131.871 \\
\hline 11 & 2016 & 10-Oct & 172.746 & 70.006 & 4900.8995 & 343094 & 24018815.86 \\
\hline \multicolumn{3}{|c|}{$\mathrm{Xr}$} & 102.739 & 0.000 & 9536.4137 & 360909 & 29278686.2 \\
\hline
\end{tabular}

$$
\begin{aligned}
\text { Sx } & =\sqrt{\frac{9536,4137}{10}}=30,881 \\
\text { Cs } & =\frac{11 \cdot 360909}{(10)(9) 30,881^{3}}=1,498 \\
\text { Ck } & =\frac{11^{2} \cdot 29278686,2}{10 \cdot 9 \cdot 8 \cdot 30,881^{4}}=5,411
\end{aligned}
$$

Because $\mathrm{Cs} \neq 0$, what is used is Log Pearson Type III 


\section{Planned Rainfall Analysis based on Log Pearson Type III}

Table 6. Log Pearson Type III Methods

\begin{tabular}{|c|c|c|c|c|c|c|c|}
\hline No & Tahun & Tanggal & $X i$ & $\log X i$ & $\log x-\log x i$ & $(\log x-\log x i) 2$ & $(\log x-\log x i) 3$ \\
\hline 1 & 2015 & 19-Mar & 71.89753 & 1.856714 & -0.139755802 & 0.019531684 & -0.002729666 \\
\hline 2 & 2007 & 8-Mar & 79.01413 & 1.897705 & -0.098764965 & 0.009754518 & -0.000963405 \\
\hline 3 & 2006 & $26 \mathrm{Peb}$ & 80.66784 & 1.9067 & -0.089769299 & 0.008058527 & -0.000723408 \\
\hline 4 & 2012 & 15-Jan & 82.16608 & 1.914693 & -0.081777195 & 0.00668751 & -0.000546886 \\
\hline 5 & 2009 & 6-Mar & 91.27208 & 1.960338 & -0.03613178 & 0.001305506 & $-4.71702 E-05$ \\
\hline 6 & 2014 & 13-Mar & 92.01767 & 1.963871 & -0.032598529 & 0.001062664 & $-3.46413 \mathrm{E}-05$ \\
\hline 7 & 2011 & 26-Dec & 98.69258 & 1.994285 & -0.002185251 & $4.77532 \mathrm{E}-06$ & $-1.04353 \mathrm{E}-08$ \\
\hline 8 & 2008 & 31-Jan & 106.1837 & 2.026058 & 0.02958829 & 0.000875467 & $2.59036 \mathrm{E}-05$ \\
\hline 9 & 2013 & 2-Jan & 109.2085 & 2.038256 & 0.041786614 & 0.001746121 & $7.29645 \mathrm{E}-05$ \\
\hline 10 & 2010 & 15-Oct & 146.265 & 2.16514 & 0.168670717 & 0.028449811 & 0.00479865 \\
\hline 11 & 2016 & 10-Oct & 172.7456 & 2.237407 & 0.240937201 & 0.058050735 & 0.013986581 \\
\hline \multicolumn{3}{|c|}{ Total } & 1130.131 & 21.96117 & 0.000000000 & 0.135527317 & 0.013838913 \\
\hline
\end{tabular}

$$
\begin{aligned}
& \log X i=\frac{21,96117}{11}=1,99647 \\
& \text { S Log X } \quad=\sqrt{\frac{0,135527317}{11-1}}=0,11641 \\
& \text { Cs } \quad=\frac{11 \cdot 0,013838}{(11-1)(11-2)(0.11641)^{3}} 1,072042
\end{aligned}
$$

Table 7. Re-period of the Log Pearson Type III method

\begin{tabular}{|r|r|r|}
\hline periode ulang & KT & RT \\
\hline 2 & -0.17499 & 94.64518 \\
\hline 5 & 0.748454 & 121.2277 \\
\hline 10 & 1.33946 & 142.038 \\
\hline 25 & 2.058309 & 172.2226 \\
\hline 50 & 2.571537 & 197.6234 \\
\hline 100 & 3.066846 & 225.6838 \\
\hline
\end{tabular}

\section{CONCLUSSION}

Flood discharge at the 25-year return period that occurred in Avfoer Sidokare's children was $450.56 \mathrm{m3} / \mathrm{sec}$, while the Afvoer capacity of Sidokare's children at residential locations with dimensions of $10 \times 4.172$ was $537,393 \mathrm{m3} / \mathrm{sec}$. Then the cross section of the Afvoer Sidokare Son does not experience overflow.

\section{REFERENCES}

Soemarto,CD. (1987_. Hidrologi Teknik. Surabaya. Usaha Nasional

Soewarno. (1995). Hidrologi aplikasi metode statistic untuk analisa data. Bandung. Nova Suripin. (2004). Sistem Drainase Perkotaan yang Berkelanjutan. ANDI Offset Yogyakarta.

Evi Nurlely (2014). Perencanaan Pengendalian Banjir Kali Krukut Jakarta (skripsi). Universitas Pendidikan Indonesia

Agantriely. N. T. 2013. Perancangan Ulang Dimensi Saluran Drainase Di Perumahan Jihad Indah Persada 2 Kecamatan Koto Tangah Padang.

Aldridge T.L. Tobing. Tinjauan Rencana Drainase di Perumahan Kota Baru Medan Hills dlam Upaya Mewujudkan Kawasan Perumahan yang Bebas Banjir

Andri Setiawan. S. 2016. Evaluasi Sistem Drainase Di Kelurahan Paminggir Garut. 14.(1)

Andy. 2009. Perencanaan Sistem Drainase Perumahan Josroyo Permai RW 11 Kecamatan Jaten Kabupaten Karanganyar [skripsi]. Surakarta (ID): Universitas Sebelas Maret.

Arifal Hidayat. 2013. Perencanaan Saluran Drainase (Studi Kasus Desa Rambah) Universitas Pasir Pengaraian 
Bambang Triadmodjo. 2008. Hidrologi Terapan. Yogya. Beta Offset

Direktorat Jendral Bina Marga DPU " Standart SK SNI m - 181989 - F. metode perhitungan debit banjir"

Fuad. 2014.Pengaruh hubungan Tata Guna Lahan Dengan Debit Banjir Pada Daerah Aliran Sungai Malalayang.4(1):45-54. 